\title{
Acute normovolemic hemodilution reduced allogeneic blood transfusion without increasing perioperative complications in patients undergoing free-flap reconstruction of the head and neck
}

\author{
Daiki Takekawa ${ }^{1}$. Junichi Saito ${ }^{1} \cdot$ Hirotaka Kinoshita ${ }^{1}$ - Eij i Hashiba ${ }^{2} \cdot$ Naoki Hirai $^{1}$ ' Yuma Yamazaki ${ }^{1}$. \\ Tetsuya Kushikata ${ }^{1} \cdot$ Kazuyoshi Hirota $^{1}$
}

Received: 3 September 2019 / Accepted: 14 November 2019 / Published online: 25 November 2019

(c) Japanese Society of Anesthesiologists 2019

\begin{abstract}
Purpose The present case-control study was conducted to evaluate whether acute normovolemic hemodilution (ANH) can reduce the need for perioperative allogeneic blood transfusion (ABT) and affect the incidence of perioperative complications in free-flap reconstruction of the head and neck.

Methods This single-center, retrospective, observational study included the perioperative data of 123 patients who underwent free-flap reconstruction of the head and neck following oncological surgery. Patients were divided into the following two groups according to whether they received ANH: ANH group and non-ANH group. We investigated whether ANH can reduce the need for perioperative ABT using propensity score-adjusted logistic regression analysis.

Results Of the 123 patients, 113 patients were assessed; 57 patients were in the ANH group and 56 patients were in the non-ANH group. The rate [ANH group vs. non-ANH group, $n(\%): 2(3.5 \%)$ vs. $23(41.1 \%), p<0.0001$ ] and amount [median (IQR): $0 \mathrm{~mL}(0,0)$ vs. $0 \mathrm{~mL}(0,280), p<0.0001]$ of ABT were significantly lower in the ANH group than in the non-ANH group. Propensity score-adjusted multivariate logistic regression analysis indicated that ANH use [odds ratio (OR): 0.040; 95\% confidence interval (CI) $0.005,0.320 ; p=0.0024)]$ was one of the independent predictors of perioperative ABT. There were no significant differences in the incidences of post-operative complications between the two groups.

Conclusion ANH use can reduce the need for perioperative ABT in patients undergoing free-flap reconstruction of the head and neck without increasing the incidence of post-operative complications.
\end{abstract}

Keywords Acute normovolemic hemodilution · Free-flap reconstruction of the head and neck · Allogeneic blood transfusion $\cdot$ Post-operative complication $\cdot$ Oncological surgery

\section{Introduction}

Perioperative allogeneic blood transfusion (ABT) is often required (12-84\%) in patients undergoing free-flap reconstruction of the head and neck following oncological surgery [1] because of the adoption of complicated procedures, including tumor resection, tracheostomy, free-flap

Daiki Takekawa

takekawa@hirosaki-u.ac.jp

1 Department of Anesthesiology, Hirosaki University Graduate School of Medicine, Hirosaki 036-8562, Japan

2 Division of Intensive Care Unit, Hiroski University Hospital, Hirosaki, Japan harvesting, and microvascular anastomosis, which are associated with significant blood loss.

Perioperative ABT can increase the risk of various complications, such as transfusion-related acute lung injury, circulatory overload, and anaphylactic reactions [2]. Perioperative $\mathrm{ABT}$ has been demonstrated to be associated with poor outcomes because of ABT-induced systemic inflammation and immune suppression [3]. Particularly, immune suppression has been reported to cause cancer recurrence and metastasis in patients undergoing oncological surgery $[4$, 5]. A retrospective cohort study demonstrated that $\mathrm{ABT}$ in patients undergoing head-and-neck free-flap surgery caused prolongation of hospital stay after surgery and caused flaprelated (hematoma and wound dehiscence), cardiac (myocardial infraction and congestive heart failure), and respiratory complications (respiratory distress and pneumonia) [6]. 
Thus, strategies to prevent or reduce $\mathrm{ABT}$ are required to improve the prognosis of cancer patients.

Acute normovolemic hemodilution (ANH) is one of the strategies to reduce the need for perioperative ABT, and it is currently being used worldwide, particularly in cardiac surgery [7]. A systematic review and meta-analysis reported that ANH has the potential to reduce the rate and amount of ABT because of less erythrocyte loss after hemodilution [8]. However, there has been no clinical investigation on the efficacy of ANH for reducing the need for perioperative ABT in free-flap reconstruction of the head and neck. Moreover, ANH reduces hematocrit during the surgical procedure and that contribute to the reduction in erythrocyte mass loss; however, hemodilution and fluid management could change the oxygen supply to the flap and post-operative course. The safety of ANH in reconstruction of the head and neck are still controversial. If ANH can reduce the rate and amount of ABT in free-flap reconstruction of the head and neck without increasing the incidence of post-operative complications, it could be an option which improves outcomes including cancer recurrence, post-operative infection, and flap success rate.

The present case-control study was performed to determine whether ANH can reduce the need for perioperative $\mathrm{ABT}$ and to evaluate whether ANH affects the incidence of perioperative complications in free-flap reconstruction of the head and neck following oncological surgery.

\section{Methods}

\section{Study procedure and patients}

This single-center, retrospective, observational study was approved by the Ethics Committee of the Hirosaki University Graduate School of Medicine, Hirosaki, Japan and was publicized on our department homepage (2019-1013). The requirement of written informed consent from the patients was waived because of the retrospective nature of the study, and the Ethics Committee approved the waiver. This study enrolled 123 patients who underwent free-flap reconstruction of the head and neck following oncological surgery at Hirosaki University Hospital between April 1, 2013 and March 31, 2019. Patients were excluded if they had preoperative anemia (hemoglobin [Hb] level less than $10.0 \mathrm{~g} /$ $\mathrm{dL}$ ), severe liver disease (Child-Pugh grade B or C), severe heart failure (New York Heart Association functional class 3 or 4 ), and end-stage kidney disease requiring intermittent hemodialysis, because these conditions met exclusion criteria for ANH. As low-volume ANH $(5-8 \mathrm{~mL} / \mathrm{kg})$ has been reported to be ineffective for reducing the need for allogeneic transfusion and post-operative bleeding [9], patients with an ANH withdrawal volume of less than $600 \mathrm{~mL}$ were excluded from the analysis. Patient characteristics and perioperative data were obtained from our hospital electronic medical and anesthesia records. Patient characteristics included sex, age, body mass index (BMI), medical history, American Society of Anesthesiologists Physical Status (ASA-PS), diagnosis, pre-operative anticancer therapy, and TNM classification of malignant tumors. Perioperative data included ANH use, flap type (osseous or non-osseous), surgery duration, intraoperative crystalloid and colloid fluid administration, intraoperative blood loss and urine output, ABT amount until discharge from the intensive-care unit (ICU), ICU stay duration, and mechanical ventilation duration. The following pre- and post-operative laboratory data were measured when patients were admitted to the ICU: Hb level, hematocrit, platelet count, blood urea nitrogen level, creatinine level, lactate level, prothrombin time, activated partial thromboplastin time, fibrinogen level, and post-operative inotrope use. Additionally, $\mathrm{Hb}$ level, hematocrit and lactate level after hemodilution, intraoperative nadir $\mathrm{Hb}$ level and hematocrit, and maximum lactate level were assessed. Post-operative complications included flap failure with impaired blood flow, post-operative bleeding requiring hemostasis, surgical site infection, acute kidney injury, atrial fibrillation, and acute heart failure. Flap failure with impaired blood flow was classified into three categories; salvaged flap failure (flap ischemia salvaged by re-microvascular surgery), partial necrosis needed debridement, and total necrosis needed re-reconstruction. Additionally, we assessed death 0-90 days and $0-180$ days after surgery. Patients were divided into the following two groups according to ANH use: ANH group and non-ANH group.

\section{Anesthesia and ANH procedure}

The principle indication for ANH in our institution is an estimated blood loss of more than $500 \mathrm{~mL}$ or a request from a surgeon for a patient with an $\mathrm{Hb}$ level of more than $10 \mathrm{~g} / \mathrm{dL}$. Exclusion criteria for ANH in our institution were uncontrolled congenital heart failure (New York Heart Association functional class 3 or 4) including active ischemic heart disease, severe liver disease (Child-Pugh grade B or C), and refusal by the patient, in addition to pre-operative anemia (Hb level less than $10 \mathrm{~g} / \mathrm{dL}$ ) and renal failure which were considered to be absolute contraindication for ANH [10]. The reason why patients with congenital heart failure including active ischemic heart disease and severe liver disease (Child-Pugh grade B or C) are excluded is that hemodilution can worsen these conditions because of reducing oxygen supply.

All surgeries were performed under general anesthesia and standard monitoring with FloTrac/Vigileo system (Edwards Lifescience, Tokyo, Japan). Even though our institution does not have specific protocol for fluid management, 
we carefully control fluid volume by referring to stroke volume variation. We usually maintain stroke volume variation between 10 and $14 \%$ after hemodilution considering reinfusion of collected blood and adjust stroke volume variation $<10 \%$ after reinfusion of collected blood. General anesthesia was induced and maintained with propofol, ketamine, remifentanil, and/or fentanyl and rocuronium. After anesthetic induction, blood was withdrawn from the central venous line, and the withdrawn blood volume for ANH was selected to avoid an $\mathrm{Hb}$ level of less than $8.0 \mathrm{~g} / \mathrm{dL}$ after hemodilution. The withdrawn blood volume was simultaneously replaced with an equal volume of $6 \%$ hydroxyethyl starch solution (130/4) (Volven; Fresenius Kabi, Bad Homburg, Germany). The collected blood was stored in a standard blood collection pack (JMS Blood Bag CPD400; JMS, Tokyo, Japan) at room temperature $\left(22-26^{\circ} \mathrm{C}\right)$ on a shaker. Arterial blood gas analysis was performed and $\mathrm{Hb}$ and lactate levels were measured immediately after hemodilution and at least every $2 \mathrm{~h}$ during surgery to evaluate oxygen delivery to peripheral tissues. When microvascular anastomosis was completed, the collected blood was reinfused to the patients.

\section{Criteria for ABT}

In our institution, the transfusion threshold is generally set at an $\mathrm{Hb}$ level of less than $7.0 \mathrm{~g} / \mathrm{dL}$. Additionally, for cases involving an increased risk of ischemia, such as cases of pulmonary disease, coronary artery disease, and cerebral vascular disease, and cases showing new electrocardiographic signs of cardiac ischemia, the transfusion threshold is set at an $\mathrm{Hb}$ level of less than $9.0 \mathrm{~g} / \mathrm{dL}$.

\section{Statistical analysis}

Patient characteristics and perioperative data are presented as median (25th-75th percentile) and number (percentage of each group). All variables were tested for normal distribution using the Kolmogorov-Smirnov test. Statistical differences between the study groups were assessed using Fisher's exact test for categorical variables and Student's $t$ test or the Mann-Whitney $U$ test for continuous variables.

Propensity score-adjusted multivariate logistic regression analysis [11] was performed to evaluate whether ANH use can reduce the need for ABT. The propensity score was the predicted probability of being in the ANH group considering the covariate values in multivariate logistic regression analysis. The selected covariates included not only age, sex, BMI, ASA-PS of $\geq 3$, pre-operative $\mathrm{Hb}$ level, and pre-operative anticancer therapy but also T3 stage or T4 stage and osseous free-flap reconstruction according to the existing knowledge that these were predictors of perioperative ABT [1]. We used the propensity score as a covariate in our model to adjust for pre-operative confounding factors. The presence of ANH was forced into the model as an explanatory variable. In addition, as intraoperative blood loss is related with perioperative $\mathrm{ABT}$, this variable was forced into the model as a predictor. It has been suggested that the number of events per predictor variable in multivariate logistic regression analysis should be at least 10 to provide an adequate predictive model [12]. However, a recent simulation study suggested that 5-9 events per predictor variable were sufficient [13]. In this study, considering the number of events ( 25 patients received $\mathrm{ABT}$ ), three variables were included in the model (one variable was included in the model for eight events). Variance inflation factor (VIF) was used to check for multicollinearity among the variables. Discrimination was measured using the area under the curve (AUC). The results are expressed as adjusted odds ratios (ORs) with corresponding 95\% confidence intervals (CIs).

All data analyses were performed using GraphPad Prism 7 (GraphPad Software Inc., San Diego, CA, USA) and EZR software version 1.27 (Saitama Medical Center, Jichi Medical University, Saitama, Japan). A $p$ value of $<0.05$ was considered statistically significant in all tests.

\section{Results}

Of the 123 enrolled patients, 113 were finally statistically analyzed (Fig. 1). All patients were estimated more than $500 \mathrm{~mL}$ of blood loss. Their characteristics are presented in Table 1. Of the 113 patients, 57 were included in the ANH group and 56 were included in the non-ANH group. There were no significant differences in sex, BMI, ASA-PS, medical history, pre-operative anticancer therapy, cancer stage, cancer type, and flap between the groups. However, age was significantly different between the groups.

The perioperative data are presented in Table 2 . In the ANH group, the ANH volume was $600 \mathrm{~mL}$ in three patients, $800 \mathrm{~mL}$ in 51 patients, and $1200 \mathrm{~mL}$ in three patients, and $\mathrm{Hb}$ level after hemodilution was $9.1 \mathrm{~g} / \mathrm{dL}(8.4,9.9)$. Among all study patients, $25(22.1 \%)$ received perioperative ABT. The rate of perioperative ABT was significantly higher in the non-ANH group (41.1\%) than in the ANH group (3.5\%) $(p<0.0001)$. Additionally, the amount of ABT was significantly lower in the ANH group than in the non-ANH group $(p<0.0001)$. The pre-operative $\mathrm{Hb}$ level and hematocrit were significantly higher in the ANH group than in the non-ANH group (13.8 vs. $12.0 \mathrm{~g} / \mathrm{dL}, p<0.0001,40.9 \%$ vs. $36.5 \%, p<0.0001$, respectively). Moreover, the postoperative $\mathrm{Hb}$ level and hematocrit remained higher in the ANH group (9.7 vs. $8.5 \mathrm{~g} / \mathrm{dL}, p<0.0001,29 \%$ vs. $26.1 \%$, $p<0.0001$, respectively). However, intraoperative blood loss was not significantly different between the groups. Although the pre-operative and post-operative lactate level were 
Fig. 1 Study population flowchart. $A N H$ acute normovolemic hemodilution

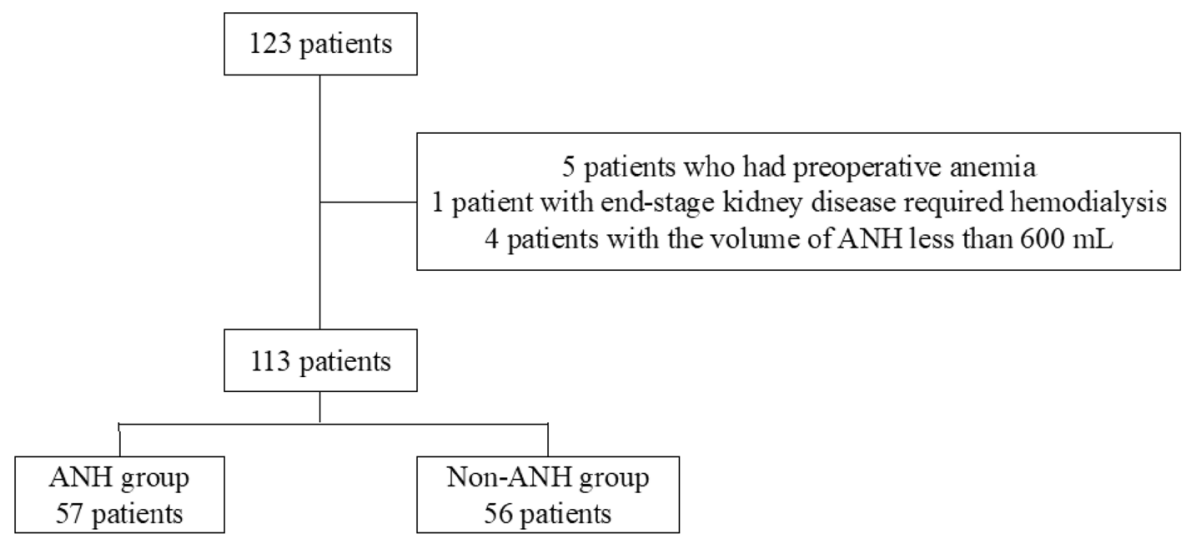

ANH: acute normovolemic hemodilution significantly higher in the ANH group than in non-ANH group, maximum lactate level was not significantly different between the groups. Moreover, changes in lactate level (postoperative lactate level - pre-operative lactate level) were not significantly different between the groups $[0.5 \mathrm{mmol} / \mathrm{L}$ $(0.1,1.0)$ vs. $0.4 \mathrm{mmol} / \mathrm{L}(0.1,0.7), p=0.3822]$. The surgery duration was significantly longer in the ANH group than in the non-ANH group $(11.6 \mathrm{~h}[10.14,12.76]$ vs. $9.8 \mathrm{~h}[8.4$, 11.7], $p=0.0008$ ). Intraoperatively, crystalloid infusion was not significantly different between the groups, but colloid infusion was significantly greater in the ANH group than in the non-ANH group (1500 vs. $1000 \mathrm{~mL}, p<0.0001)$. In addition, there were no significant differences in the incidences of post-operative complications between the groups (Table 3).

The propensity score-adjusted multivariate logistic regression analysis revealed that ANH use (OR: 0.040, 95\% CI $0.005,0.320 ; p=0.0024$ ) and intraoperative blood loss (per $100 \mathrm{~mL}$ increase, OR: 1.400, 95\% CI 1.160, 1.700; $p=0.0006)$ were independently associated with perioperative ABT (Table 4), suggesting that ANH use can reduce the need for perioperative ABT. No VIF value was up to 10 , indicating that there was no collinearity in the model. The AUC value was 0.894 (95\% CI 0.829, 0.959).

\section{Discussion}

The present study found that ANH can reduce the need for perioperative $\mathrm{ABT}$ and does not increase the incidence of perioperative complications in reconstruction of the head and neck. To the best of our knowledge, this is the first study to elucidate the efficacy of ANH for reducing the need for perioperative $\mathrm{ABT}$ and to assess the safety of $\mathrm{ANH}$ in reconstruction of the head and neck.

Although some background variables, such as age and pre-operative $\mathrm{Hb}$ level, significantly differed between the
$\mathrm{ANH}$ and non-ANH groups, the rate and amount of $\mathrm{ABT}$ were significantly lower in the ANH group than in the nonANH group. The propensity score-adjusted multivariate logistic regression analysis indicated that ANH use and intraoperative blood loss were independent factors associated with perioperative ABT. Additionally, there were no significant differences in the incidences of post-operative complications between the two groups. Therefore, ANH can reduce the need for perioperative $\mathrm{ABT}$ without increasing the risk of post-operative complications.

Although no previous studies have evaluated the efficacy of ANH for reducing the need for perioperative ABT in patients undergoing free-flap reconstruction of the head and neck, the findings of some previous studies in other surgical settings support our positive findings [7, 8, 14, 15]. A previous meta-analysis of randomized trials revealed that $\mathrm{ANH}$ reduced both the rate and amount of ABT in cardiac surgery [8]. Additionally, a retrospective study showed that ANH was an effective approach to reduce the need for ABT in patients undergoing gynecological cancer surgery [14]. Moreover, a prospective randomized-controlled study demonstrated that ANH reduced the rate of perioperative ABT (10\% vs. 36\%) in hepatectomy patients [15]. The present data showed that $\mathrm{ANH}$ could reduce the need for $\mathrm{ABT}$ in free-flap reconstruction of the head and neck following oncological surgery, which involves a long duration.

One of the concerns about ANH use is post-operative complications related with increased intraoperative fluid administration [16]. A previous randomized trial revealed that ANH use significantly increased anastomotic site complications without reducing the need for ABT when compared with standard patient management [16]. The authors mentioned that these results were likely associated with greater intraoperative fluid administration in the $\mathrm{ANH}$ group than in the non-ANH group (8000 vs. $6000 \mathrm{~mL}$ ). In contrast, the present study revealed that ANH use did not increase the incidence of perioperative complications. This might 
Table 1 Patient characteristics

\begin{tabular}{|c|c|c|c|}
\hline & ANH group & Non-ANH group & $p$ value \\
\hline$n$ & 57 & 56 & \\
\hline Male & $40(70.2 \%)$ & $30(53.6 \%)$ & 0.0828 \\
\hline Age (year) & $65(55,69.5)$ & $72(65,78)$ & $<0.0001 *$ \\
\hline $\operatorname{BMI}\left(\mathrm{kg} / \mathrm{m}^{2}\right)$ & $22.3(20.5,25.2)$ & $21.1(18.8,24.4)$ & 0.0743 \\
\hline ASA-PS & & & 0.2642 \\
\hline 1 or 2 & $47(82.5 \%)$ & $41(73.2 \%)$ & \\
\hline 3 & $10(17.5 \%)$ & $15(26.8 \%)$ & \\
\hline \multicolumn{4}{|l|}{ Medical history } \\
\hline Hypertension & $22(38.6 \%)$ & $31(55.4 \%)$ & 0.0909 \\
\hline $\mathrm{DM}$ & $7(12.3 \%)$ & $9(16.1 \%)$ & 0.6001 \\
\hline Dyslipidemia & $9(15.8 \%)$ & $6(10.7 \%)$ & 0.5808 \\
\hline COPD & $5(8.8 \%)$ & $6(10.7 \%)$ & 0.7616 \\
\hline Stroke & $4(7.0 \%)$ & $3(5.4 \%)$ & $>0.9999$ \\
\hline IHD & $1(1.8 \%)$ & $4(7.1 \%)$ & 0.2062 \\
\hline Risk of ischemia & $5(8.8 \%)$ & $5(8.9 \%)$ & $>0.9999$ \\
\hline \multicolumn{4}{|c|}{ Pre-operative anticancer therapy } \\
\hline NAC & $2(3.5 \%)$ & $8(14.3 \%)$ & 0.0529 \\
\hline PRT & $5(8.8 \%)$ & $6(10.7 \%)$ & 0.7616 \\
\hline Type of cancer & & & 0.1450 \\
\hline Oral cavity & $46(80.7 \%)$ & $50(89.3 \%)$ & \\
\hline Oropharynx & $3(5.3 \%)$ & $3(5.4 \%)$ & \\
\hline Hypopharynx & $3(5.3 \%)$ & $2(3.6 \%)$ & \\
\hline Others & $5(8.8 \%)$ & $1(1.8 \%)$ & \\
\hline $\mathrm{T}$ stage & & & 0.2110 \\
\hline I or II & $26(45.6 \%)$ & $33(58.9 \%)$ & \\
\hline III or IV & $27(47.3 \%)$ & $22(39.3 \%)$ & \\
\hline Unknown & $4(7.0 \%)$ & $1(1.8 \%)$ & \\
\hline $\mathrm{N}$ stage & & & 0.7660 \\
\hline 0 & $29(50.9 \%)$ & $25(44.6 \%)$ & \\
\hline I & $12(21.1 \%)$ & $12(21.4 \%)$ & \\
\hline II & $14(24.6 \%)$ & $18(32.1 \%)$ & \\
\hline Unknown & $2(3.5 \%)$ & $1(1.8 \%)$ & \\
\hline M stage & & & $>0.9999$ \\
\hline 0 & $57(100 \%)$ & $56(100 \%)$ & \\
\hline I & $0(0 \%)$ & $0(0 \%)$ & \\
\hline Type of flap & & & 0.4897 \\
\hline Osseous & $3(5.3 \%)$ & $5(8.9 \%)$ & \\
\hline Non-osseous & $54(94.7 \%)$ & $51(91.1 \%)$ & \\
\hline
\end{tabular}

Differences between the ANH and non-ANH groups were estimated using Fisher's exact test for categorical variables and Student's $t$ test or the Mann-Whitney $U$ test for continuous variables. Data are presented as number (percentage of each group) or median (25th-75th percentile)

$A N H$ acute normovolemic hemodilution, BMI body mass index, ASA$P S$ American Society of Anesthesiologists Physical Status, DM diabetes mellitus, COPD chronic obstructive pulmonary disease, $I H D$ ischemic heart disease, NAC neoadjuvant chemotherapy, $P R T$ preoperative radiotherapy

*Statistical significance be associated with restrictive fluid management to prevent fluid overload during the perioperative period. In the present study, although intraoperative colloid infusion was significantly greater in the ANH group than in the non-ANH group, intraoperative crystalloid infusion did not differ between the groups. A previous meta-analysis of randomized-controlled trials demonstrated that perioperative goal-directed hemodynamic therapy, including restricted fluid management, could reduce the incidence of post-operative complications in major surgery [17]. Therefore, careful fluid management with reduced crystalloid infusion might avoid preventable complications.

In the univariate analysis, there was no significant difference in flap failure between the ANH and non-ANH groups. It has been reported that acute isovolemic reduction of the $\mathrm{Hb}$ level to $5.0 \mathrm{~g} / \mathrm{dL}$ in conscious healthy resting humans does not show evidence of inadequate systemic oxygen delivery, as assessed by the lack of changes in oxygen consumption and the plasma lactate level [18]. In our institution, as the blood withdrawal volume for ANH was selected to avoid an $\mathrm{Hb}$ level of less than $8.0 \mathrm{~g} / \mathrm{dL}$ after hemodilution and arterial blood gas analysis, Hb-level assessment and lactate-level assessment were performed at least every $2 \mathrm{~h}$ during surgery, ANH was conducted without an increase in the incidence of post-operative complications. In the present study, $\mathrm{Hb}$ level after hemodilution was more than $8.0 \mathrm{~g} / \mathrm{dL}$ and maximum lactate level was not significantly different between the groups. However, the ideal $\mathrm{Hb}$ level for tissue oxygenation of a free flap remains unclear. Indeed, the lack of oxygen carriers during excessive hemodilution might cause hypoxic damage in surgical flaps [19]. In contrast, moderate hemodilution is known to facilitate microcirculatory blood flow in ischemic flaps by decreasing vascular resistance [20]. An animal experimentation study revealed that ANH could improve oxygenation in ischemic and hypoxic flap tissues [21]. Moreover, in a previous study, there were no differences in flap-related and medical complications between transfusion triggers of hematocrit less than $21 \%$ versus hematocrit less than $27 \%$ in free-flap reconstruction of the head and neck [22]. Thus, the present level of ANH for free-flap surgery was likely not to increase flap failure.

In the present study, there was no significant difference in intraoperative blood loss between the ANH and non-ANH groups. A previous study revealed that ANH resulted in a hypocoagulable state, but the change in clotting ability was slight, and this could not affect the amount of intraoperative blood loss [23]. Indeed, there was no significant difference in post-operative bleeding requiring hemostasis in the present study. Although the withdrawn whole blood was stored in a standard blood collection pack at room temperature $\left(22-26^{\circ} \mathrm{C}\right)$ on a shaker for more than approximately $8 \mathrm{~h}$, the post-operative platelet count and coagulation ability 
Table 2 Perioperative data of the patients

\begin{tabular}{|c|c|c|c|}
\hline & Group ANH & Group non-ANH & $p$ value \\
\hline \multicolumn{4}{|l|}{ Perioperative Labo data } \\
\hline \multicolumn{4}{|l|}{$\mathrm{Hb}(\mathrm{g} / \mathrm{dL})$} \\
\hline Pre & $13.8(13.0,14.8)$ & $12.0(10.9,13.1)$ & $<0.0001^{*}$ \\
\hline After hemodilution & $9.1(8.4,9.9)$ & - & - \\
\hline Intra. Nadir & $8.2(7.2,8.9)$ & $8.0(6.8,9.0)$ & 0.5923 \\
\hline Post & $9.7(8.9,10.7)$ & $8.5(7.8,9.3)$ & $<0.0001 *$ \\
\hline \multicolumn{4}{|l|}{ Hematocrit (\%) } \\
\hline Pre & $40.9(38.8,43.6)$ & $36.5(32.9,38.6)$ & $<0.0001^{*}$ \\
\hline After hemodilution & $27.1(25.0,28.3)$ & - & - \\
\hline Intra. Nadir & $24.3(21.7,25.8)$ & $24.1(20.3,26.5)$ & 0.5630 \\
\hline Post & $29.0(27,32.3)$ & $26.1(23.9,27.9)$ & $<0.0001 *$ \\
\hline \multicolumn{4}{|l|}{ Plt $\left(\times 10^{4} / \mu \mathrm{L}\right)$} \\
\hline Pre & $19.6(16.7,25.0)$ & $19.0(14.9,23.5)$ & 0.3100 \\
\hline Post & $14.5(11.0,16.8)$ & $12.8(9.7,17.4)$ & 0.7689 \\
\hline \multicolumn{4}{|l|}{ BUN (mg/dL) } \\
\hline Pre & $12(11,16)$ & $14(11,16)$ & 0.1436 \\
\hline Post & $9(8,11)$ & $10(8,13)$ & 0.1420 \\
\hline \multicolumn{4}{|l|}{ Cre (mg/dL) } \\
\hline Pre & $0.71(0.60,0.88)$ & $0.77(0.64,0.93)$ & 0.3911 \\
\hline Post & $0.74(0.61,0.88)$ & $0.72(0.60,0.87)$ & 0.6835 \\
\hline \multicolumn{4}{|l|}{$\mathrm{Lac}(\mathrm{mmol} / \mathrm{L})$} \\
\hline Pre & $0.9(0.7,1.3)$ & $0.8(0.6,1.0)$ & $0.0054 *$ \\
\hline After hemodilution & $1.0(0.7,1.1)$ & - & - \\
\hline Intra. Max & $1.3(1.0,1.6)$ & $1.2(0.9,1.6)$ & 0.3038 \\
\hline Post & $1.5(1.0,2.0)$ & $1.1(0.8,1.6)$ & $0.0151 *$ \\
\hline \multicolumn{4}{|l|}{$\mathrm{PT}(\mathrm{s})$} \\
\hline Pre & $13.6(12.6,14.4)$ & $14.0(12.2,14.5)$ & 0.5765 \\
\hline Post & $16.2(14.9,16.7)$ & $15.9(13.5,17.3)$ & 0.9404 \\
\hline \multicolumn{4}{|l|}{ APTT (s) } \\
\hline Pre & $29.3(27.8,30.7)$ & $29.2(27.3,31.5)$ & 0.9774 \\
\hline Post & $35.2(32.3,39.3)$ & $36.3(33.1,39.2)$ & 0.3939 \\
\hline \multicolumn{4}{|l|}{ Fib (mg/dL) } \\
\hline Pre & $333(293,393)$ & $365(290,465)$ & 0.2150 \\
\hline Post & $288(227,326)$ & $296(215,354)$ & 0.6708 \\
\hline Duration of surgery (h) & $11.6(10.1,12.8)$ & $9.8(8.4,11.7)$ & $0.0008 *$ \\
\hline Intra. Bleeding $(\mathrm{mL})$ & $390(282,525)$ & $465(300,608)$ & 0.2226 \\
\hline Intra. Bleeding $(\mathrm{mL} / \mathrm{kg})$ & $6.6(4.7,9.4)$ & $7.9(5.5,11.6)$ & 0.0776 \\
\hline Intra. UO (ml/kg/h) & $2.9(2.3,4.0)$ & $3.4(2.0,4.4)$ & 0.5157 \\
\hline \multicolumn{4}{|l|}{ Intra. infusion } \\
\hline Total $(\mathrm{ml} / \mathrm{kg} / \mathrm{h})$ & $7.5(6.0,9.1)$ & $7.4(5.9,7.8 .8)$ & 0.6606 \\
\hline Crystalloid (ml/kg/h) & $5.4(4.2,7.2)$ & $6.1(4.3,7.7)$ & 0.2760 \\
\hline Colloid (ml) & $1500(1225,2000)$ & $1000(500,1350)$ & $<0.0001 *$ \\
\hline \multicolumn{4}{|l|}{ Intra. and post. $\mathrm{ABT}$} \\
\hline $\mathrm{RBC}(\mathrm{mL})$ & $0(0,0)$ & $0(0,280)$ & $<0.0001^{*}$ \\
\hline $\mathrm{RBC}(\mathrm{mL} / \mathrm{kg})$ & $0(0,0)$ & $0(0,5.9)$ & $<0.0001^{*}$ \\
\hline $\operatorname{RBC}(\mathrm{N})$ & $2(3.5 \%)$ & $23(41.1 \%)$ & $<0.0001^{*}$ \\
\hline FFP $(\mathrm{mL})$ & $0(0,0)$ & $0(0,0)$ & 0.2434 \\
\hline $\mathrm{FFP}(\mathrm{mL} / \mathrm{kg})$ & $0(0,0)$ & $0(0,0)$ & 0.2434 \\
\hline $\operatorname{FFP}(n)$ & $0(0 \%)$ & $2(3.6 \%)$ & 0.4963 \\
\hline $\mathrm{PC}(\mathrm{mL})$ & $0(0,0)$ & $0(0,0)$ & $>0.9999$ \\
\hline $\mathrm{PC}(\mathrm{mL} / \mathrm{kg})$ & $0(0,0)$ & $0(0,0)$ & $>0.9999$ \\
\hline $\mathrm{PC}(n)$ & $0(0 \%)$ & $0(0 \%)$ & $>0.9999$ \\
\hline \multicolumn{4}{|c|}{ Intra. and post. use of inotropes } \\
\hline Naradrenaline $(n)$ & $39(68.4 \%)$ & $41(73.2 \%)$ & 0.6800 \\
\hline Landiolol $(n)$ & $10(17.5 \%)$ & $6(10.7 \%)$ & 0.4194 \\
\hline Duration of & & & \\
\hline
\end{tabular}


Table 2 (continued)

\begin{tabular}{lccc}
\hline & Group ANH & Group non-ANH & $p$ value \\
\hline ICU stay (day) & $4(3,4.5)$ & $4(4,5)$ & 0.0566 \\
Ventilator (day) & $1(1,1)$ & $1(1,1)$ & 0.8797 \\
\hline
\end{tabular}

Differences between the ANH and non-ANH groups were estimated using Fisher's exact test for categorical variables and Student's $t$ test or the Mann-Whitney $U$ test for continuous variables. Data are presented as number (percentage of each group) or median (25th-75th percentile)

$A N H$ acute normovolemic hemodilution, Pre pre-operative, Intra intraoperative, Post post-operative, $\mathrm{Hb}$ hemoglobin, Plt platelet count, BUN blood urea nitrogen, Cre creatinine, Lac Lactate, PT prothrombin time, $A P T T$ activated partial thromboplastin time, Fib fibrinogen, $U O$ urine output, $A B T$ allogeneic blood transfusion, $R B C$ red blood cell, $F F P$ fresh-frozen plasma, $P C$ platelet concentrate, $I C U$ intensive-care unit

*Statistical significance

Table 3 Post-operative complications

\begin{tabular}{llll}
\hline & Group ANH & Group non-ANH & $p$ value \\
\hline Over all & $8(14.0 \%)$ & $10(17.9 \%)$ & 0.6161 \\
Surgical & $7(12.3 \%)$ & $8(14.3 \%)$ & 0.7883 \\
Flap failure with & & & 0.2600 \\
$\quad$ impaired blood flow & & & \\
Salvaged flap failure & $1(1.8 \%)$ & $1(1.8 \%)$ & \\
Partial necrosis & $0(0 \%)$ & $3(5.4 \%)$ & \\
Total necrosis & $5(8.8 \%)$ & $2(3.6 \%)$ & \\
Post. bleeding & $0(0 \%)$ & $2(3.6 \%)$ & 0.2434 \\
SSI & $1(1.8 \%)$ & $2(3.6 \%)$ & 0.6183 \\
Medical & $2(3.5 \%)$ & $3(5.4 \%)$ & 0.6790 \\
AKI & $2(3.5 \%)$ & $1(1.8 \%)$ & $>0.9999$ \\
Af & $0(0 \%)$ & $2(3.6 \%)$ & 0.2434 \\
AHF & $0(0 \%)$ & $1(1.8 \%)$ & 0.4956 \\
Death within & & & \\
0-90 days & $0(0 \%)$ & $1(1.8 \%)$ & 0.4956 \\
0-180 days & $1(1.8 \%)$ & $2(3.6 \%)$ & 0.6183 \\
\hline
\end{tabular}

Differences between the ANH and non-ANH groups were estimated using Fisher's exact test. Data are presented as number (percentage of each group)

ANH acute normovolemic hemodilution, Post post-operative, SSI surgical site infection, $A K I$ acute kidney injury, $A f$ atrial fibrillation, $A H F$ acute heart failure

*Statistical significance

were not significantly different between the two groups. A previous study reported that storage of whole blood for $24 \mathrm{~h}$ at room temperature resulted in a $23 \%$ decrease in the activity of Factor VIII, but caused no significant decrease in the activities of coagulation Factor V, Factor VII, Factor XI, Factor XII, fibrinogen, antithrombin, and von Willebrand factor [24]. This study concluded that storage of whole blood at ambient temperature for $24 \mathrm{~h}$ has a minimal effect on the coagulation activity of plasma and that this approach is an acceptable alternative to producing plasma on the day of blood collection. Thus, ANH in prolonged surgery might not increase perioperative blood loss.
Table 4 Propensity score-adjusted multivariate logistic regression model to identify the predictive factors of perioperative ABT

\begin{tabular}{llll}
\hline & OR & $95 \%$ CI & $p$ value \\
\hline ANH & 0.040 & $0.005,0.320$ & $0.0024^{*}$ \\
Intra. Bleeding (per $100 \mathrm{ml})$ & 1.400 & $1.160,1.700$ & $0.0006^{*}$ \\
Propensity score & 0.464 & $0.054,3.970$ & 0.4830 \\
\hline
\end{tabular}

The propensity score was estimated using multivariate logistic regression analysis with the following variables: age, sex, body mass index, American Society of Anesthesiologists Physical Status 3 or more, pre-operative hemoglobin level, T3 or T4 stages, osseous free-flap reconstruction, neoadjuvant chemotherapy, and pre-operative radiotherapy. The propensity score was 0.49 (25th-75th percentile: 0.19 and 0.83 ). ANH use, intraoperative blood loss, and the propensity score were included in this model. No variance inflation factor value was up to 10 , indicating that there was no collinearity in the model. The area under the curve was 0.894 (95\% CI: 0.829, 0.959)

$A B T$ allogeneic blood transfusion, $A N H$ acute normovolemic hemodilution, $O R$ odds ratio, $C I$ confidence interval, Intra. Intraoperative

*Statistical significance

The present study has several limitations. First, this was a single-center, retrospective, observational study and the sample size was relatively small. There might have been selection bias and undetected confounding factors that affected the results. Thus, the results should be treated with caution. Second, other intraoperative hemodynamic parameters, such as mixed venous blood oxygen saturation and cardiac output, were not recorded. Measurement of these variables would have allowed evaluation of the relationships between the variables and post-operative complications, including flap failure. Third, although we have investigated mortality within 90 days and 180 days after surgery, patients were not followed with regard to ABT and complications after discharge from the ICU. Thus, we could not evaluate the long-term outcomes of ANH, including 5-year survival rate as cancer prognosis, in free-flap reconstruction of the head and neck.

In conclusion, our study showed that ANH use can reduce the need for perioperative ABT without increasing the incidence of post-operative complications in patients undergoing 
free-flap reconstruction of the head and neck following oncological surgery.

Funding None.

\section{Compliance with ethical standards}

Conflict of interest The authors declare that they have no conflict of interest.

\section{References}

1. Shah MD, Goldstein DP, McCluskey SA, Miles BA, Hofer SO, Brown DH, Irish JC, Gullane PJ, Gilbert RW. Blood transfusion prediction in patients undergoing major head and neck surgery with free-flap reconstruction. Arch Otolaryngol Head Neck Surg. 2013;139:771.

2. Hendrickson JE, Roubinian NH, Chowdhury D, Brambilla D, Murphy EL, Wu Y, Ness PM, Gehrie EA, Snyder EL, Hauser RG, Gottschall JL, Kleinman S, Kakaiya R, Strauss RG, National Heart, Lung, and Blood Institute (NHLBI) Recipient Epidemiology, and Donor Evaluation Study (REDS-III). Incidence of transfusion reactions: a multi-center study utilizing systematic active surveillance and expert adjudication. Transfusion. 2016;56:2587-96.

3. Remy KE, Hall MW, Cholette J, Juffermans NP, Nicol K, Doctor A, Blumberg N, Spinella PC, Norris PJ, Dahmer MK, Muszynski JA, Pediatric Critical Care Blood Research Network (Blood Net). Mechanisms of red blood cell transfusion-related immunomodulation. Transfusion. 2018;58:804-15.

4. Wu HL, Tai YH, Lin SP, Chan MY, Chen HH, Chang KY. The impact of blood transfusion on recurrence and mortality following colorectal cancer resection: a propensity score analysis of 4,030 patients. Sci Rep. 2018;8:13345.

5. Abu-Ghanem Y, Dotan Z, Zilberman DE, Kaver I, Ramon J. Intraoperative but not postoperative blood transfusion adversely affect cancer recurrence and survival following nephrectomy for renal cell carcinoma. Sci Rep. 2019;9:1160.

6. Puram SV, Yarlagadda BB, Sethi R, Muralidhar V, Chambers KJ, Emerick KS, Rocco JW, Lin DT, Deschler DG. Transfusion in head and neck free flap patients: practice patterns and a comparative analysis by flap type. Otolaryngol Head Neck Surg. 2015;152:449-57.

7. Zhou X, Zhang C, Wang Y, Yu L, Yan M. Preoperative Acute normovolemic hemodilution for minimizing allogeneic blood transfusion: a meta-analysis. Anesth Analg. 2015;121:1443-555.

8. Barile L, Fominskiy E, Di Tomasso N, Alpìzar Castro LE, Landoni G, De Luca M, Bignami E, Sala A, Zangrillo A, Monaco F. Acute normovolemic hemodilution reduces allogeneic blood cell transfusion in cardiac surgery: a systematic review and metaanalysis of randomized trials. Anesth Analg. 2017;124:743-52.

9. Casati V, Speziali G, D'Alessandro C, Cianchi C, Antonietta Grasso M, Spagnolo S, Sandrelli L. Intraoperative low-volume acute normovolemic hemodilution in adult open-heart surgery. Anesthesiology. 2002;97:367-73.
10. Stehling L, Zauder HL. Acute normovolemic hemodilution. Transfusion. 1991;31:857-68.

11. Jason SH, Roger JL. The propensity score. JAMA. 2015;314:1637-8.

12. Peduzzi P, Concato J, Kemper E, Holford TR, Feinstein AR. A simulation study of the number of events per variable in logistic regression analysis. J Clin Epidemiol. 1996;49:1373-9.

13. Vittinghoff E, McClloch CE. Relaxing the rule of ten events per variable in logistic and Cox regression. Am J Epidemiol. 2007; 165:710-8.

14. Saito J, Masui K, Noguchi S, Nakai K, Tamai Y, Midorikawa Y, Kinoshita H, Mikami N, Kitayama M, Hashimoto H, Hirota K. The efficacy of acute normovolemic hemodilution for preventing perioperative allogeneic blood transfusion in gynecological cancer patients. J Clin Anesth. 2020;60:42-3.

15. Matot I, Scheinin O, Jurim O, Eid A. Effectiveness of acute normovolemic hemodilution to minimize allogeneic blood transfusion in major liver resections. Anesthesiology. 2002;97:794-800.

16. Fischer M, Matsuo K, Gonen M, Grant F, Dematteo RP, D’Angelica MI, Mascarenhas J, Brennan MF, Allen PJ, Blumgart LH, Jarnagin WR. Relationship between intraoperative fluid administration and perioperative outcome after pancreaticoduodenectomy: results of a prospective randomized trial of acute normovolemic hemodilution compared with standard intraoperative management. Ann Surg. 2010;252:952-8.

17. Giglio MT, Marucci M, Testini M, Brienza N. Goal-directed haemodynamic therapy and gastrointestinal complications in major surgery: a meta-analysis of randomized controlled trials. Br J Anaesth. 2009;103:637-46.

18. Weiskopf RB, Viele MK, Feiner J, Kelley S, Lieberman J, Noorani M, Leung JM, Fisher DM, Murray WR, Toy P, Moore MA. Human cardiovascular and metabolic response to acute, severe isovolemic anemia. JAMA. 1998;279:217-21.

19. Sigurdsson GH. Perioperative fluid management in microvascular surgery. J Reconstr Microsurg. 1995;11:57-655.

20. Barker JH, Hammersen F, Galla TJ, Bondàr I, Zeller P, Menger MD, Messmer K. Direct monitoring of capillary perfusion following normovolemic hemodilution in an experimental skin-flap model. Plast Reconstr Surg. 1990;86:946-54.

21. Schramm S, Wettstein R, Wessendorf R, Jakob SM, Banic A, Erni D. Acute normovolemic hemodilution improves oxygenation in ischemic flap tissue. Anesthesiology. 2002;96:1478-84.

22. Abt NB, Puram SV, Sinha S, Sethi RKV, Goyal N, Emerick KS, Lin DT, Deschler DG. Transfusion in head and neck cancer patients undergoing pedicled flap reconstruction. Laryngoscope. 2018;128:E409-E415415.

23. Shin HJ, Na HS, Do SH. The effects of acute normovolaemic haemodilution on peri-operative coagulation in total hip arthroplasty. Anaesthesia. 2015;70:304-9.

24. Cardigan R, Van der Meer PF, Pergande C, Cookson P, BaumannBaretti B, Cancelas JA, Devine D, Gulliksson H, Vassallo R, de Wildt-Eggen J. Coagulation factor content of plasma produced from whole blood stored for 24 hours at ambient temperature: results from an international multicenter BEST Collaborative study. Transfusion. 2011;51(Suppl 1):50S-S5757.

Publisher's Note Springer Nature remains neutral with regard to jurisdictional claims in published maps and institutional affiliations. 